bioRxiv preprint doi: https://doi.org/10.1101/2022.02.03.478984; this version posted February 3, 2022. The copyright holder for this preprint (which was not certified by peer review) is the author/funder, who has granted bioRxiv a license to display the preprint in perpetuity. It is made available under aCC-BY 4.0 International license.

\title{
Activating ligands of Uncoupling protein 1 identified by rapid membrane protein thermostability shift analysis
}

Riccardo Cavalieria, Marlou Klein Hazebroek ${ }^{b}$, Camila A. Cotrima, Yang Lee ${ }^{\dagger \dagger}$, Edmund R. S. Kunjic, Martin Jastroch ${ }^{\mathrm{b}}$, Susanne Keipert ${ }^{\mathrm{b}}$ and Paul G. Crichton ${ }^{\mathrm{a} 1}$

${ }^{a}$ Biomedical Research Centre, Norwich Medical School, University of East Anglia, Norwich Research Park, Norwich NR4 7TJ, United Kingdom

${ }^{b}$ Department of Molecular Biosciences, The Wenner-Gren Institute, Stockholm University, SE-106 91, Stockholm, Sweden

${ }^{c}$ Medical Research Council, Mitochondrial Biology Unit, University of Cambridge, Cambridge Biomedical
Campus, Hills Road, Keith Peters Building, CB2 OXY, United Kingdom

${ }^{\dagger}$ Current address: Laboratory of Molecular Biology, Medical Research Council, Cambridge Biomedical Campus, Francis Crick Avenue, Cambridge CB2 OQH, United Kingdom

Author contributions:

R.C. and P.G.C. designed research and wrote the paper; R.C., M.K.H., C.A.C., S.K. and P.G.C. performed research; M.J., Y.L. and E.R.S.K. contributed materials and expertise; R.C., M.K.H., C.A.C., M.J., S.K. and P.G.C. analyzed data; all authors contributed to editing

The authors declare no conflict of interest

${ }^{1}$ To whom correspondence may be addressed. E-mail: P.Crichton@uea.ac.uk

Keywords: ligand binding; thermal stability assay; differential scanning fluorimetry; brown adipose tissue; proton transport; energy expenditure; mitochondrial carrier

Short title: Ligands to activate UCP1 thermogenesis

\begin{abstract}
Uncoupling protein 1 (UCP1) catalyzes mitochondrial proton leak in brown adipose tissue to facilitate nutrient oxidation for heat production, and its activation may counteract obesity and metabolic disease in humans. The protein is activated by free fatty acids, which are generated in brown adipocytes following adrenergic stimulation (e.g. in response to cold exposure) to induce proton leak by UCP1 through an unclear interaction. Here, we have characterized activator binding to purified UCP1 by protein thermostability shift analysis, which, unlike many conventional approaches, can inform on the binding of hydrophobic ligands to membrane proteins. We show that fatty acids and other activators influence UCP1 through a specific destabilizing interaction, which can be distinguished from general denaturation induced by these ionic detergent-like species in tests. The degree of destabilization correlates with activator potency, as confirmed in liposome activity assays, and matches UCP1 destabilization induced by equivalent alkyl sulfonates that are transported by the protein, consistent with activators interacting as transport substrates that shift the carrier to a less stable conformation of the transport cycle. Through the detection of stability shifts in screens, we identify novel activators, including the over-the-counter drug ibuprofen, where ligand analysis indicates a relatively wide structural specificity for interacting molecules. Ibuprofen induces UCP1 activity in liposomes, isolated brown fat mitochondria and UCP1-expressing HEK293 cells, but not in cultured brown adipocytes, suggesting that the targeting of UCP1 in cells by approved drugs is in principle achievable as a therapeutic avenue, but requires variants with more effective delivery in brown adipocytes.
\end{abstract}




\section{Significance Statement}

Inducing thermogenesis by Uncoupling protein 1 (UCP1) activity in brown fat is a promising strategy to combat obesity and diabetes. However, the adrenergic stimulation of the tissue lacks specificity, adversely impacting health, and targeting UCP1 directly is hampered by a poorly understood interaction of the protein with fatty acid activators. Here, we have investigated ligand binding to purified UCP1 through rapid thermostability shift analysis, and show that activating molecules interact to shift UCP1 to a less stable conformation, binding as transport substrates. These effectors can be readily distinguished from other ligands, allowing the detection of novel species in screens. The identification of varied activators including the drug ibuprofen opens up therapeutic avenues for directly targeting UCP1 for activation in cells.

\section{Introduction}

Brown adipose tissue of mammals has the specialized ability to oxidise nutrients to generate heat for defence of body temperature against the cold (1), and may help combat metabolic disease in humans. Brown fat occurrence in adults correlates with leanness (2-4) where activation of the tissue increases energy expenditure, lipid turnover, glucose disposal and insulin sensitivity, consistent with a positive impact on systemic metabolism and health (5-8). Thermogenesis by brown fat is achieved through the activation of Uncoupling protein 1 (UCP1), a $33 \mathrm{kDa}$ mitochondrial carrier that catalyses the leak of protons across the mitochondrial inner membrane, dissipating the protonmotive force to release energy as heat at the expense of ATP synthesis (see (9) for review). Following the adrenergic stimulation of brown adipocytes, e.g. by norepinephrine following cold exposure, intracellular cAMPdependent signalling initiates lipolysis and the release of long chain fatty acids, which directly interact to activate UCP1, overcoming the inhibition of the protein by cytosolic purine nucleotides (10). Methods to encourage brown fat development and the activation of UCP1 in the absence of physiological stimuli is a therapeutic strategy to combat diabetes, obesity and related diseases, e.g., by pharmacological activation of adipocyte $\beta_{3}$-adrenergic receptors $(6$, 11).

How fatty acids induce proton leak by UCP1 is debated (see (9) for biochemical models). Fatty acid anions may act as a co-factor to complete a proton transport channel within the protein (12) or, instead, act as a transport substrate that is exported by UCP1 to flip back directly across the mitochondrial inner membrane in a protonated form, independent of the protein, to give a net proton transfer $(13,14)$. Alternatively, fatty acids in both protonated and deprotonated forms may be transported by UCP1, where long chain species remain bound to the protein following transport across the membrane, allowing just protons to be chaperoned down their electrochemical gradient (15). The fatty acids may also compete directly or act allosterically to remove inhibitory purine nucleotides, which bind to UCP1 from the cytosolic side (16).

UCP1 is a member of the mitochondrial carrier family of metabolite transporters, which all share the same basic fold and membrane topology. They are comprised of three $\sim 100$ amino acid repeat domains, each composed of two transmembrane $\alpha$-helices linked by a matrix loop and small $\alpha$-helix, which together form a six transmembrane helix barrel arrangement with three-fold pseudosymmetry $(17,18)$. Our purification studies of native UCP1 have revealed that the protein is a monomer and tightly binds three cardiolipin molecules to maintain stability, similar to other mitochondrial carriers (19). Though to what degree UCP1 employs a common carrier metabolite transport mechanism, as recently resolved for the ADP/ATP carrier (20), for proton leak is not clear. The protein has been postulated to employ a mechanistically distinct process for proton leak (21). UCP1 has been studied for many years, yet the location and molecular nature of the fatty acid binding site has not been clarified. For membrane proteins in general, there has been a lack of efficient methods to report on ligand interactions due to the practical complications imposed by membrane hydrophobicity and common need for detergents. Fatty acids add further challenges as ionic surfactants, binding non-specifically with potential to denature proteins. Information on fatty acid activator binding to UCP1 has 
largely been indirect and inferred from changes in nucleotide binding or activity measurements (e.g. $(16,22,23))$. An improved understanding of the activator interaction may provide therapeutic avenues to target UCP1 directly for activation to engage thermogenesis.

Here, we demonstrate the efficient detection of activator interactions with purified UCP1 through protein thermal stability shift analysis. The approach not only informs on the nature of the interaction process but provides an effective screening avenue to identify novel molecules with potential to activate UCP1 in cells.

\section{Results}

UCP1 thermostability shifts detect a specific destabilizing interaction with activators. We have applied a rapid fluorescence-based assay to assess the relative thermostability of purified UCP1, which can provide practical information on membrane protein integrity and interaction with detergent, lipid and ligands (24). Samples in the assay are subjected to a temperature ramp while protein unfolding is monitored by an increase in fluorescence of the probe $\mathrm{N}$-[4-(7-diethylamino-4-methyl-3-coumarinyl)phenyl]maleimide (CPM), which reacts with protein thiols as they become solvent exposed due to denaturation to give a fluorescent adduct (cf. (25)). When assessed, UCP1 purified in dodecyl maltose neopentyl glycol (12MNG) detergent exhibited a background fluorescence consistent with at least one solventexposed cysteine residue present in the native state, with a transition to a higher plateau as remaining buried cysteines are revealed during protein unfolding (Fig. 1A, top), as observed previously in similar detergents $(19,24)$. Our sequencing of the ovine UCP1 gene coding region verified the presence of 8 cysteine residues in the protein (Fig. S1; rather than 9 reported in wider sequencing data elsewhere, e.g. (26)). The peak in the derivative of the unfolding transition provides an apparent 'melt temperature' $\left(T_{\mathrm{m}}\right)$ as a relative measure of thermal stability for a given protein population (24) (e.g. $51.0^{\circ} \mathrm{C}$ for UCP1, Fig. 1A, bottom). This measure relates to the strength and sum of the molecular bonding that occurs within and between associated components, which contribute to the overall protein stability. Accordingly, we have found the apparent $T_{\mathrm{m}}$ of membrane proteins to exhibit distinct shifts $\left(\Delta T_{\mathrm{m}}\right)$ in the presence of ligands, consistent with reporting on the bonding changes associated with ligand interaction $(19,24,27)$.

GDP increases the stability of UCP1 (e.g. with a $\Delta T_{\mathrm{m}}$ of $+15^{\circ} \mathrm{C}$ at $1 \mathrm{mM}$, Fig1A, bottom), where larger stabilisation is observed in conditions where the inhibitor is known to bind more tightly (cf. (24)). In contrast, when tested, we found that the UCP1 activator oleic acid destabilized UCP1 $\left(\Delta T_{\mathrm{m}}\right.$ of $-6^{\circ} \mathrm{C}$ at $25 \mu \mathrm{M}$, Fig. $1 \mathrm{~A}$, bottom). Fatty acids can act as harsh ionic soap at neutral $\mathrm{pH}$ and potentially denature proteins non-specifically. Importantly, however, when the oleic acid concentration was varied, the drop in $T_{\mathrm{m}}$ did not magnify as a simple function of the concentration, as would be expected of a non-specific effect, but instead plateaued towards a $\Delta T_{\mathrm{m}}$ of $-6^{\circ} \mathrm{C}$ between 20 and $40 \mu \mathrm{M}$ oleic acid (Fig. 1B), consistent with saturation of a specific interaction event that results in net decreased bonding within the UCP1 population. Above $\sim 40 \mu \mathrm{M}$, a second less consistent phase was observed with an increased drop in stability with oleic acid concentration, likely representing the saturation of the species in the proteindetergent micelles and the onset of generic protein denaturation.

Further tests with other known activators of UCP1 revealed similar trends, consistently showing a destabilizing effect. The UCP1 activators retinoic acid and TTNPB (cf. $(28,29)$ ) both destabilized UCP1 with a saturating behaviour when assessed over similar concentrations (towards a maximum $\Delta T_{\mathrm{m}}$ of $\sim-3^{\circ} \mathrm{C}$, Fig. 1C), as did the long chain fatty acid nonadecanoic acid (C19; towards a $\Delta T_{\mathrm{m}} \sim-4^{\circ} \mathrm{C}$, Fig 1D). The shorter chain and less potent activator of UCP1 dodecanoic acid (C12) also destabilized UCP1 but with smaller $\Delta T_{\mathrm{m}}$ shifts and without a distinguishable saturation profile, whereas the shortest fatty acid tested, heptanoic acid (C7), showed almost no destabilization at all (Fig. 1D and E). Notably, the size of the shift observed had a strong correlation with fatty acid length and ability to activate UCP1, as verified by proton leak activity assays with UCP1 proteoliposomes (cf. Fig. 1F and 1E). These thermostability measurements reveal that a specific destabilization of purified UCP1 is 
bioRxiv preprint doi: https://doi.org/10.1101/2022.02.03.478984; this version posted February 3, 2022. The copyright holder for this preprint (which was not certified by peer review) is the author/funder, who has granted bioRxiv a license to display the preprint in perpetuity. It is made available under aCC-BY 4.0 International license.

fundamental to the interaction with activators, and suggests a net reduction in total bonding occurs in the protein population relating to the activation process.

Activators interact with UCP1 as transport substrates. Ligand-protein binding generates new bonds, hence, an apparent decrease in overall bonding suggests significant conformational changes occur elsewhere in the protein to account for bond loss, such as those that occur in transporter proteins to facilitate substrate translocation (cf. (30)). UCP1 has been proposed to transport fatty acids in some mechanistic models of proton leak (see above), which is supported by the observed transport of the fatty acid analogues alkyl sulfonates by the protein $(13,15,31)$. In contrast to fatty acids, these species cannot be protonated at physiological $\mathrm{pH}$, having very low pKa values $(\sim-2)$, and retain a negative charge that prevents them from diffusing directly across lipid membranes. These anions are transported by UCP1 but, importantly, they do not activate proton leak by the protein $(13,15,31)$. When tested, we found that they induced almost identical trends in UCP1 thermostability to their equivalent fatty acid counterparts. Similar to C19, octadecane sulfonate (C18-S) destabilized the protein, saturating towards a $\Delta T_{\mathrm{m}}$ of $\sim-6^{\circ} \mathrm{C}$ between 20 and $40 \mu \mathrm{M}$ (Fig. 2A). Likewise, the shorter chain variants, undecane sulfonate (C11-S) and hexane sulfonate (C6-S) showed smaller shifts, matching $\mathrm{C} 12$ and $\mathrm{C} 7$, respectively, where the overall amplitude of the change observed correlated with chain length (Fig. $2 A$ and $B$ ). In further tests with an alternative long chain fatty acid analogue, oleoyl-L- $\alpha$-lysophosphatidic acid (OLPA), an inhibitor of UCP1 activity (15)), only minor shifts were observed in contrast to C18-S and C19 (Fig. 2C). These correlations indicate that activators destabilize UCP1 by interacting in an identical manner to transport substrates, rather than as activators of proton conductance per se, and that fatty acid anion transport is fundamental to the activation process. Of significance, the related ADP/ATP carrier was also found to be destabilized by micromolar concentrations of transport substrate (ADP) in similar assay conditions (24), suggesting a common substrate interaction process in both of these carriers.

Novel activators of proton leak are found among compounds that destabilize UCP1. In light of the distinct stability changes detected with UCP1 activators, we used thermostability shift assays to screen a further 72 selected compounds, including fatty acid analogues, metabolites and drugs, to identify interacting species of potential significance (Fig. S2). This approach identified 20 compounds that induced a significant $\Delta T_{\mathrm{m}}$ in UCP1, 16 that were destabilizing and four that were stabilizing (Fig. 3A). Nine of the compounds that destabilized have been reported previously as activating ligands of proton leak or anions transported by $\operatorname{UCP} 1(15,22,32,33)$, or were drugs of the retinoid class (adapalene, acitretin and tazarotene) related to the known UCP1 activator retinoic acid (29). Whereas one of the compounds that stabilized, mant-GDP, a nucleotide derivative, is reported to inhibit UCP1 (23). The remaining 10 compounds represent potential novel ligands of UCP1.

Compounds of interest were tested in UCP1 proteoliposome assays to determine their impact on proton leak activity. Strikingly, five of the destabilizing species tested proved to be activators of UCP1-mediated proton leak, as indicated by significantly increased proton conductance rates relative to controls, which were sensitive to GDP (Fig. 3B). In contrast, none of the compounds tested that either increased or had no effect on protein stability activated proton leak activity by UCP1. Nor did any of the non-nucleotide stabilizing ligands show significant inhibition of oleic acid-induced proton leak by UCP1 in follow up tests (Fig. S3). These trends corroborate that the changes in UCP1 responsible for destabilization are likely an essential feature of activation. Importantly, the process identified three new compounds as direct activating ligands of the UCP1 protein: tetradecylthioacetic acid, a $\beta$ oxidation-resistant synthetic fatty acid that activates peroxisome proliferator-activated receptors (PPAR) and promotes mitochondrial fatty acid oxidation (34); TUG-891, a G protein coupled receptor 120 (GPR120) agonist reported to stimulate UCP1-dependent mitochondrial uncoupling, fat oxidation and weight loss in mice (35); and ibuprofen, a widely used nonsteroidal anti-inflammatory drug and painkiller (36). 
All of the UCP1-activating species observed were hydrophobic $(\log P>3.5)$ but amphipathic in nature, with a carboxylate head group that could be protonated at physiological $\mathrm{pH}$ to facilitate proton transport $\left(p K_{a}>4\right)$, consistent with past observations $(15,22)$. However, the bulk hydrophobic region varied considerably from long chain fatty acids across activators (e.g. TUG-891), suggesting a lack of specific structural constraints. Computational alignment and analysis of verified activators revealed common matching features associated with the carboxylate headgroup for potential polar interactions but no specific features across the bulk region other than general hydrophobicity (see Fig. S4A). The wider array of UCP1 ligands identified by thermostability analysis alone exhibited similar characteristics but with added diversity in the polar region and no strict requirement for a high-p $K_{a}$ carboxylate group (see Fig. S4B). These findings indicate that UCP1 has a particularly broad ligand specificity, which widens the scope of molecules with potential to activate the protein.

Ibuprofen activates UCP1 activity in isolated brown adipose tissue mitochondria and
HEK293 cells but not in immortalized brown adipocytes. Our screening process revealed that the licenced analgesic ibuprofen can activate isolated UCP1. Further investigations with the more soluble metabolic derivates indicated that only the parent molecule activated the protein in liposomes (Fig. S5A), in line with the wider observed physiochemical trends. Tests with isolated mitochondria from mouse brown adipose tissue confirmed that ibuprofen could activate UCP1 in the natural membrane environment. Similar to palmitate, the drug decreased the membrane potential of succinate-energized mitochondria from WT mice in a GDPsensitive manner, but not in mitochondria from UCP1 KO mice (see tests of safranin fluorescence quenching by mitochondria, Fig. 4A to C: with no activator, palmitate or ibuprofen addition, respectively). We tested the ability of ibuprofen to activate UCP1 in a cellular environment using both immortalized brown adipocytes from mice and a HEK293 cell UCP1expression system (28). Whilst respiratory analysis did not indicate a stimulation of UCP1dependent respiration in brown adipocytes (Fig. S5 B and C), tests with HEK293 cells transfected with mouse UCP1 (MmUCP1) revealed a dose-dependent stimulation of nonphosphorylating respiration by $\geq 50 \mu \mathrm{M}$ ibuprofen specific to UCP1-expressing cells (cf. oligomycin-insensitive oxygen consumption rates in UCP1-expressing vs empty vector control cells, Fig 4D-F). The drug was able to induce UCP1-dependent oxygen consumption similar to the TTNPB control, albeit requiring higher concentrations ( $500 \mu \mathrm{M}$ vs $15 \mu \mathrm{M}$ TTNPB, cf. Fig. 4D-F). These findings provide a proof of principal for our process of identifying novel ligands that have the potential to activate UCP1-dependent energy expenditure in cells. The contrasting lack of response in cultured brown adipocytes suggests that other factors prevent the action of the drug on UCP1 in these cells, such as restricted uptake or a more rapid turnover to inactive variants. Circumventing these factors, e.g. via the development of suitable compound variants, therefore, is a promising new area for further research.

\section{Discussion}

Integral membrane proteins like UCP1 are challenging to study. Hydrophobic ligands, such as fatty acids, can bind non-specifically and potentially act as harsh ionic detergents that denature proteins, hampering binding studies. Furthermore, UCP1 and other mitochondrial carriers are relatively unstable and can be extracted in detergent in an incorrectly folded form, leading to erroneous conclusions on function or biophysical properties (e.g. from common bacterial expression systems; see $(24,37-41)$ and references therein). Here, we have utilized a fluorescence-based protein thermostability assay tailored for membrane proteins to reveal details on the interaction of purified UCP1 with activators. The method reports on stability shifts related to the bonding changes of the protein itself, informing on interacting ligands and potential protein conformational shifts, avoiding issues of conventional binding studies. The methodology is emerging as a useful tool in resolving structural mechanisms and clarifying substrates in other carriers and transport proteins $(20,24,27,42,43)$. Inherent to the technique is the monitoring of the folded state of the protein, which provides a robust indicator of protein sample integrity. Our experimental approach here not only reveals mechanistic 
bioRxiv preprint doi: https://doi.org/10.1101/2022.02.03.478984; this version posted February 3, 2022. The copyright holder for this preprint (which was not certified by peer review) is the author/funder, who has granted bioRxiv a license to display the preprint in perpetuity. It is made available under aCC-BY 4.0 International license.

information on how activators influence native UCP1, but also that the protein has a wide ligand specificity and may be directly targeted, independent of fatty acids, for increased energy expenditure.

Our thermostability measurements consistently demonstrated a destabilizing interaction of proton leak activators with purified UCP1, which matched the changes observed by equivalent alkyl sulfonate anions that do not facilitate proton leak but are transport substrates of the protein. The common destabilization profiles suggest the same bonding changes occur and that fatty acids and other activators interact as transport substrates, and that activator transport is fundamental in the UCP1 proton conductance mechanism. As such, our binding study observations support biochemical models of UCP1 proton leak where the protein specifically acts to transport the activating species $(13,15)$ over other models (see $(9)$ ). Accordingly, UCP1 exports bound fatty acid anions across the mitochondrial inner membrane, where they return to the matrix in a protonated form, either via UCP1 (shuttling model (15)) or by independently flipping directly across the membrane (cycling model (13)) to give a net proton conductance. In the former case, long chain fatty acid species are proposed to remain bound to UCP1 during transport in either direction, essentially chaperoning protons. Of the compounds that we observed to destabilize UCP1 that likely represent transport substrates, a subset proved to be activators. Notably, these all had $\mathrm{p} K_{\mathrm{a}}$ values $>4$, consistent with the notion that it is the inherent ability of the molecule to be protonated in the relevant $\mathrm{pH}$ conditions that dictates whether or not a transported species facilitates proton leak, in line with previous evidence in support of these models $(15,22)$.

The distinct loss in stability from activator binding also provides clues to the structural process underlying UCP1 proton leak. The related mitochondrial ATPIADP carrier exchanges nucleotides by a recently clarified structural mechanism, which is likely conserved across the mitochondrial carrier family of metabolite transporters (20). The protein cycles between a cytoplasmic (c-state) and matrix (m-state) conformation to alternate access of a substrate binding site within the central cavity of the protein to either side of the membrane for stepwise substrate transport. Substrate-binding induced state shifts occur through symmetrical movements in the three pseudo-symmetrically related domains of the carrier. Notably, deviations from symmetry occur in the central binding site to accommodate the nonsymmetrical nature of the substrate. Similar to our observations here with UCP1, the purified ATP/ADP carrier was also destabilized by micromolar concentrations of ADP substrate, or by the m-state inhibitor bongkrekic acid, in similar conditions (24), consistent with ligand-induced transitioning to a less stable state of the transport cycle. The similar net bonding loss in UCP1 suggests that activating 'substrates' induce similar state shifts, and that activation uses the same core transport mechanism rather than a distinct asymmetrical process, as proposed (21, 44), where fatty acid anions translocate on the outside surface of the protein at the lipid-protein interface. UCP1 conserves all of the key structural elements of a conventional carrier mechanism, with no distinct asymmetrical features at the membrane-facing surface to facilitate specific interactions for ligand translocation (9), supporting this rationale. The region in UCP1 corresponding to the substrate binding site of common carriers (see (45)) has a triplet of arginine residues known to influence nucleotide binding $(46,47)$, which have potential to interact with the polar/charged group of activators as well, where hydrophobic residues in the central cavity could accommodate additional hydrophobic interactions. Notably, fatty acid activators and nucleotides have been reported to display competitive behaviour in regulating UCP1 (16).

Our screening approach successfully identified novel activating ligands among compounds that destabilized UCP1. The synthetic GPR120 agonist TUG-891 promotes brown adipose tissue thermogenic activity in mice through GPR120-signalling, but is also reported to induce UCP1-dependent oxygen consumption in isolated mitochondria, suggesting an additional mechanism (35). Our results corroborate these claims and demonstrate that TUG-891 is a direct activating ligand of the UCP1 protein, despite its structural deviation from conventional fatty acid activators. The positive health attributes observed in mice with this compound (increased fat oxidation, reduces body weight and fat mass) are at least in part likely due to the direct targeting of UCP1. The non- $\beta$-oxidizable fatty acid analogue, tetradecylthioacetic 
acid, which promotes mitochondrial fatty acid oxidation through PPAR activation (34), may also confer health benefits as a hypolipidemic agent via UCP1 targeting given our findings, though this notion requires verification. Surprisingly, we discovered that the cyclooxygenase inhibitor and common painkiller ibuprofen is an activating ligand of UCP1 as well, and could activate the protein in isolated brown fat mitochondria, HEK293 cells but not brown adipocytes. Ibuprofen may be sequestered and/or metabolized in brown adipocytes (e.g. by triglyceride incorporation, as can occur in white adipocytes (48)) that, if rapid enough, could prevent it from activating UCP1. Alternatively, the drug may not be taken up by these cells in contrast to kidney cell lines where uptake has been demonstrated to be mediated by an unknown transporter (49). Compound variants circumventing such barriers, therefore, are an attractive avenue for further investigation.

There is considerable research into methods to encourage brown fat proliferation and the browning of white adipose tissue, though in the absence of physiological stimuli, UCP1 must still be activated to benefit from the full glucose and triglyceride turnover capacity of the tissue (50). Pharmacological activation of upstream beta-adrenergic targets has been demonstrated in humans but lacks tissue specificity and is complicated by wider systemic effects $(6,51)$. As a specific, defining feature of brown fat, UCP1 is an attractive target for the therapeutic activation of thermogenic energy expenditure. Our assessment utilizing thermostability shift analysis provides an effective route to probe for UCP1-interacting molecules and reveals that the protein can be directly activated by conventional drug-like compounds with relatively wide structural specificity, which opens up the array of candidate molecules with potential to target it.

\section{Materials and Methods}

Details on gene sequencing, mitochondrial isolation, native UCP1 purification, protein thermostability shift measurements, liposome reconstitution and proton flux measurements, mammalian cell culture methods, membrane potential measurements, extracellular flux respiratory measurements and Western blotting can be found in the SI Materials and Methods.

\section{Acknowledgements}

We would like to thank local Norfolk and Suffolk farms for access to new-born lambs that died of natural causes during the lambing season. This work was supported a PhD studentship award (R.C.) from Norwich Medical School and the Faculty of Medicine and Health, University of East Anglia, UK, and by funding from the UK Biological and Biotechnological Sciences Research Council (BB/S00940X/1), Novo Nordisk Research Foundation (0059646 to M.J.) and Swedish Research Council (2018-02150 to S.K).

\section{Bibliography}

1. B. Cannon, J. Nedergaard, Brown adipose tissue: function and physiological significance. Physiol Rev 84, 277-359 (2004).

2. T. Yoneshiro et al., Age-related decrease in cold-activated brown adipose tissue and accumulation of body fat in healthy humans. Obesity (Silver Spring) 19, 1755-1760 (2011).

3. A. M. Cypess et al., Identification and importance of brown adipose tissue in adult humans. N Engl J Med 360, 1509-1517 (2009).

4. J. Nedergaard, T. Bengtsson, B. Cannon, Unexpected evidence for active brown adipose tissue in adult humans. Am J Physiol Endocrinol Metab 293, E444-452 (2007).

5. M. Chondronikola et al., Brown adipose tissue improves whole-body glucose homeostasis and insulin sensitivity in humans. Diabetes 63, 4089-4099 (2014). 
bioRxiv preprint doi: https://doi.org/10.1101/2022.02.03.478984; this version posted February 3, 2022. The copyright holder for this preprint (which was not certified by peer review) is the author/funder, who has granted bioRxiv a license to display the preprint in perpetuity. It is made available under aCC-BY 4.0 International license.

6. A. M. Cypess et al., Activation of human brown adipose tissue by a beta3-adrenergic receptor agonist. Cell Metab 21, 33-38 (2015).

7. M. Saito et al., High incidence of metabolically active brown adipose tissue in healthy adult humans: effects of cold exposure and adiposity. Diabetes 58, 1526-1531 (2009).

8. M. Chondronikola et al., Brown Adipose Tissue Activation Is Linked to Distinct Systemic Effects on Lipid Metabolism in Humans. Cell Metab 23, 1200-1206 (2016).

9. P. G. Crichton, Y. Lee, E. R. Kunji, The molecular features of uncoupling protein 1 support a conventional mitochondrial carrier-like mechanism. Biochimie 134, 35-50 (2017).

10. R. M. Locke, E. Rial, I. D. Scott, D. G. Nicholls, Fatty acids as acute regulators of the proton conductance of hamster brown-fat mitochondria. Eur J Biochem 129, 373-380 (1982).

11. A. M. Singh et al., Human beige adipocytes for drug discovery and cell therapy in metabolic diseases. Nat Commun 11, 2758 (2020).

12. M. Klingenberg, S. G. Huang, Structure and function of the uncoupling protein from brown adipose tissue. Biochim Biophys Acta 1415, 271-296 (1999).

13. K. D. Garlid, D. E. Orosz, M. Modriansky, S. Vassanelli, P. Jezek, On the mechanism of fatty acid-induced proton transport by mitochondrial uncoupling protein. J Biol Chem 271, 2615-2620 (1996).

14. K. D. Garlid, M. Jaburek, P. Jezek, The mechanism of proton transport mediated by mitochondrial uncoupling proteins. FEBS Lett 438, 10-14 (1998).

15. A. Fedorenko, P. V. Lishko, Y. Kirichok, Mechanism of fatty-acid-dependent UCP1 uncoupling in brown fat mitochondria. Cell 151, 400-413 (2012).

16. I. G. Shabalina, A. Jacobsson, B. Cannon, J. Nedergaard, Native UCP1 displays simple competitive kinetics between the regulators purine nucleotides and fatty acids. J Biol Chem 279, 38236-38248 (2004).

17. E. Pebay-Peyroula et al., Structure of mitochondrial ADP/ATP carrier in complex with carboxyatractyloside. Nature 426, 39-44 (2003).

18. H. Aquila, T. A. Link, M. Klingenberg, Solute carriers involved in energy transfer of mitochondria form a homologous protein family. FEBS Lett 212, 1-9 (1987).

19. Y. Lee, C. Willers, E. R. Kunji, P. G. Crichton, Uncoupling protein 1 binds one nucleotide per monomer and is stabilized by tightly bound cardiolipin. Proc Natl Acad Sci U S A 112, 6973-6978 (2015).

20. J. J. Ruprecht et al., The Molecular Mechanism of Transport by the Mitochondrial ADPIATP Carrier. Cell 176, 435-447.e415 (2019).

21. P. Jezek, M. Jaburek, K. D. Garlid, Channel character of uncoupling proteinmediated transport. FEBS Lett 584, 2135-2141 (2010).

22. P. Jezek, M. Modriansky, K. D. Garlid, A structure-activity study of fatty acid interaction with mitochondrial uncoupling protein. FEBS Lett 408, 166-170 (1997).

23. A. S. Divakaruni, D. M. Humphrey, M. D. Brand, Fatty acids change the conformation of uncoupling protein 1 (UCP1). J Biol Chem 287, 36845-36853 (2012).

24. P. G. Crichton et al., Trends in thermostability provide information on the nature of substrate, inhibitor, and lipid interactions with mitochondrial carriers. J Biol Chem 290, 8206-8217 (2015).

25. A. I. Alexandrov, M. Mileni, E. Y. Chien, M. A. Hanson, R. C. Stevens, Microscale fluorescent thermal stability assay for membrane proteins. Structure 16, 351-359 (2008).

26. Y. N. Yuan, W. Z. Liu, J. H. Liu, L. Y. Qiao, J. L. Wu, Cloning and ontogenetic expression of the uncoupling protein 1 gene UCP1 in sheep. J App/ Genet 53, 203$212(2012)$.

27. H. Majd et al., Screening of candidate substrates and coupling ions of transporters by thermostability shift assays. Elife 7 (2018).

28. S. Keipert, M. Jastroch, Brite/beige fat and UCP1 - is it thermogenesis? Biochim Biophys Acta 1837, 1075-1082 (2014). 
29. E. Rial et al., Retinoids activate proton transport by the uncoupling proteins UCP1 and UCP2. Embo j 18, 5827-5833 (1999).

30. M. Klingenberg, Ligand-protein interaction in biomembrane carriers. The induced transition fit of transport catalysis. Biochemistry 44, 8563-8570 (2005).

31. P. Jezek, K. D. Garlid, New substrates and competitive inhibitors of the Cltranslocating pathway of the uncoupling protein of brown adipose tissue mitochondria. J Biol Chem 265, 19303-19311 (1990).

32. E. Winkler, M. Klingenberg, Effect of fatty acids on $\mathrm{H}+$ transport activity of the reconstituted uncoupling protein. J Biol Chem 269, 2508-2515 (1994).

33. I. G. Shabalina, E. C. Backlund, J. Bar-Tana, B. Cannon, J. Nedergaard, Within brown-fat cells, UCP1-mediated fatty acid-induced uncoupling is independent of fatty acid metabolism. Biochim Biophys Acta 1777, 642-650 (2008).

34. B. Bjørndal, L. Burri, V. Staalesen, J. Skorve, R. K. Berge, Different adipose depots: their role in the development of metabolic syndrome and mitochondrial response to hypolipidemic agents. J Obes 2011, 490650 (2011).

35. M. Schilperoort et al., The GPR120 agonist TUG-891 promotes metabolic health by stimulating mitochondrial respiration in brown fat. EMBO Mol Med 10 (2018).

36. K. D. Rainsford, Ibuprofen: pharmacology, efficacy and safety. Inflammopharmacology 17, 275-342 (2009).

37. M. S. King, P. G. Crichton, J. J. Ruprecht, E. R. S. Kunji, Concerns with yeast mitochondrial ADP/ATP carrier's integrity in DPC. Nat Struct Mol Biol 25, 747-749 (2018).

38. M. S. Piel, S. Masscheleyn, F. Bouillaud, K. Moncoq, B. Miroux, Structural models of mitochondrial uncoupling proteins obtained in DPC micelles are not functionally relevant. Febs j 10.1111/febs.15629 (2020).

39. V. Kurauskas et al., How Detergent Impacts Membrane Proteins: Atomic-Level Views of Mitochondrial Carriers in Dodecylphosphocholine. J Phys Chem Lett 9, 933-938 (2018).

40. F. Dehez, P. Schanda, M. S. King, E. R. S. Kunji, C. Chipot, Mitochondrial ADP/ATP Carrier in Dodecylphosphocholine Binds Cardiolipins with Non-native Affinity. Biophys J 113, 2311-2315 (2017).

41. M. Zoonens et al., Dangerous liaisons between detergents and membrane proteins. The case of mitochondrial uncoupling protein 2. J Am Chem Soc 135, 15174-15182 (2013).

42. M. S. King, M. Kerr, P. G. Crichton, R. Springett, E. R. Kunji, Formation of a cytoplasmic salt bridge network in the matrix state is a fundamental step in the transport mechanism of the mitochondrial ADP/ATP carrier. Biochim Biophys Acta 1857, 14-22 (2016).

43. S. P. Harborne, M. S. King, P. G. Crichton, E. R. Kunji, Calcium regulation of the human mitochondrial ATP-Mg/Pi carrier SLC25A24 uses a locking pin mechanism. Sci Rep 7, 45383 (2017).

44. P. Ježek, M. Jabůrek, R. K. Porter, Uncoupling mechanism and redox regulation of mitochondrial uncoupling protein 1 (UCP1). Biochim Biophys Acta Bioenerg 1860, 259-269 (2019).

45. A. J. Robinson, E. R. Kunji, Mitochondrial carriers in the cytoplasmic state have a common substrate binding site. Proc Natl Acad Sci U S A 103, 2617-2622 (2006).

46. M. Modriansky, D. L. Murdza-Inglis, H. V. Patel, K. B. Freeman, K. D. Garlid, Identification by site-directed mutagenesis of three arginines in uncoupling protein that are essential for nucleotide binding and inhibition. J Biol Chem 272, 2475924762 (1997).

47. M. Klingenberg, K. S. Echtay, Uncoupling proteins: the issues from a biochemist point of view. Biochim Biophys Acta 1504, 128-143 (2001).

48. S. Vickery, P. F. Dodds, Incorporation of xenobiotic carboxylic acids into lipids by cultured 3T3-L1 adipocytes. Xenobiotica 34, 1025-1042 (2004). 
49. C. U. Nielsen et al., A Transporter of Ibuprofen is Upregulated in MDCK I Cells under Hyperosmotic Culture Conditions. Mol Pharm 13, 3119-3129 (2016).

50. J. Nedergaard, B. Cannon, The changed metabolic world with human brown adipose tissue: therapeutic visions. Cell Metab 11, 268-272 (2010).

51. A. S. Baskin et al., Regulation of Human Adipose Tissue Activation, Gallbladder

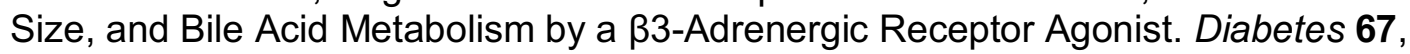
2113-2125 (2018).

Abbreviations: UCP1, Uncoupling protein 1; 12MNG, dodecyl maltose neopentyl glycol (2,2didecylpropane-1,3-bis- $\beta$-D-maltopyranoside); OLPA, oleoyl-L- $\alpha$-lysophosphatidic acid; TTNPB, 4-[(E)-2-(5,6,7,8-Tetrahydro-5,5,8,8-tetramethyl-2-naphthalenyl)-1-propenyl]benzoic acid; C7, heptanoic acid; C12, dodecanoic acid; C19, nonadecanoic acid; C6-S, hexane sulfonate; C11-S, undecane sulfonate; C18-S, octadecane sulfonate

\section{Figures and Legends}


A.

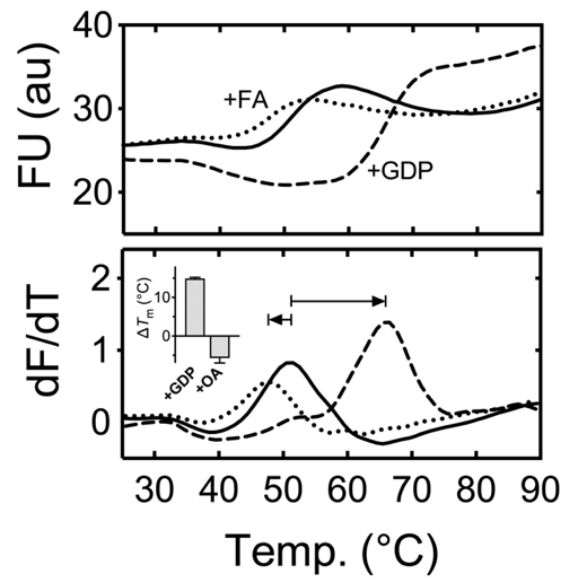

C.
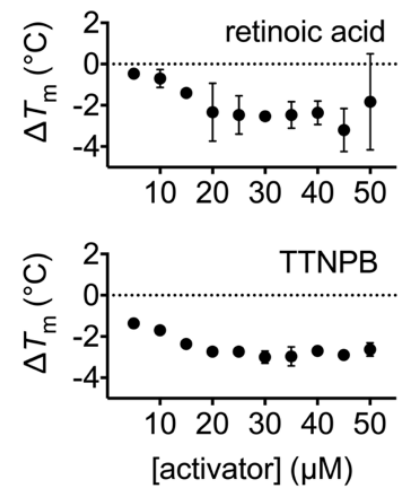

E.

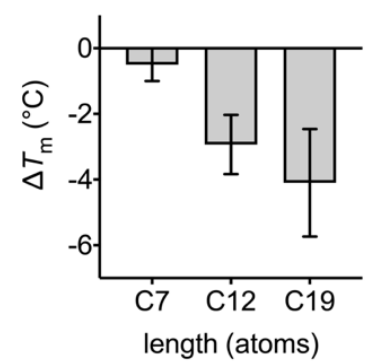

B.

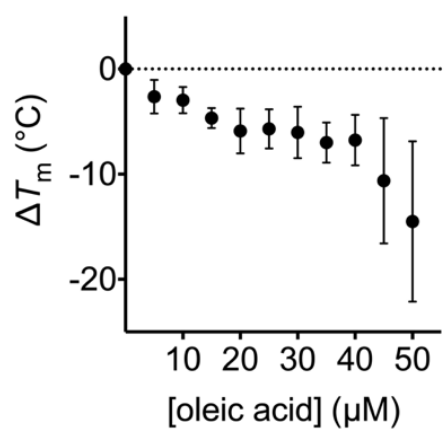

D.
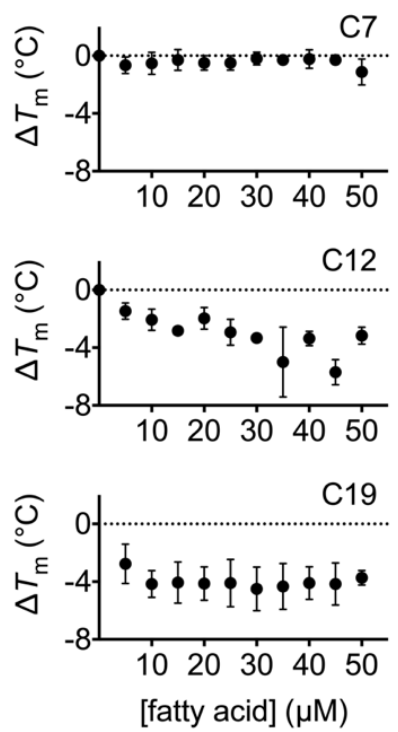

$\mathrm{F}$.

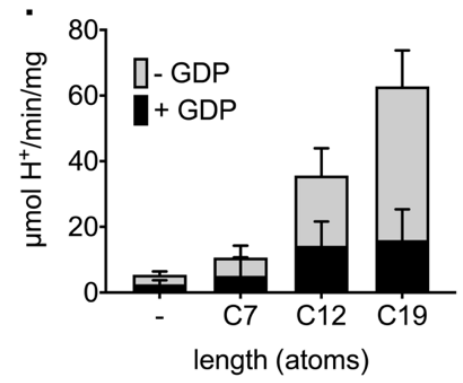

Figure 1. Activators induce a specific destabilization of native UCP1. The relative thermal stability of purified UCP1 monitored by the fluorescence of CPM-adduct formation at cysteine residues as they become solvent-exposed due to thermal denaturation (see Methods). (A) The thermal denaturation profile (top), and corresponding first derivative (bottom), of native UCP1 in assay buffer with $0.1 \% 12 \mathrm{MNG}$ detergent in the absence (solid line) or presence of 1 $\mathrm{mM}$ GDP (dashed line) or $25 \mu \mathrm{M}$ oleic acid (dotted line). Inset, average ( \pm SD) shift in UCP1 'melt temperature' ( $\Delta T_{\mathrm{m}}$; condition minus control) associated with either ligand. (B-D) Corresponding shifts in UCP1 thermal stability associated with the addition of the indicated concentration of oleic acid (B), retinoid activators $(C)$ or saturated fatty acids of differing carbon alkyl chain length (D, and $E$, at $25 \mu \mathrm{M}$ specifically; $C 7$, heptanoic acid; $C 12$, dodecanoic acid; C19, nonadecanoic acid). (F) The corresponding proton uptake rates ( $\mathrm{nmol} \mathrm{H} \mathrm{H}^{+/} \mathrm{min} / \mathrm{mg}$ protein) by UCP1 reconstituted into liposomes in the absence or presence of $100 \mu \mathrm{M} \mathrm{C7,C12}$ or C19, with and without $1 \mathrm{mM}$ GDP, as indicated. Data are averages ( $\pm S D)$ of 3-4 independent experiments for $\Delta T_{\mathrm{m}}$ values and 4 independent experiments for proton uptake rates. 
A.
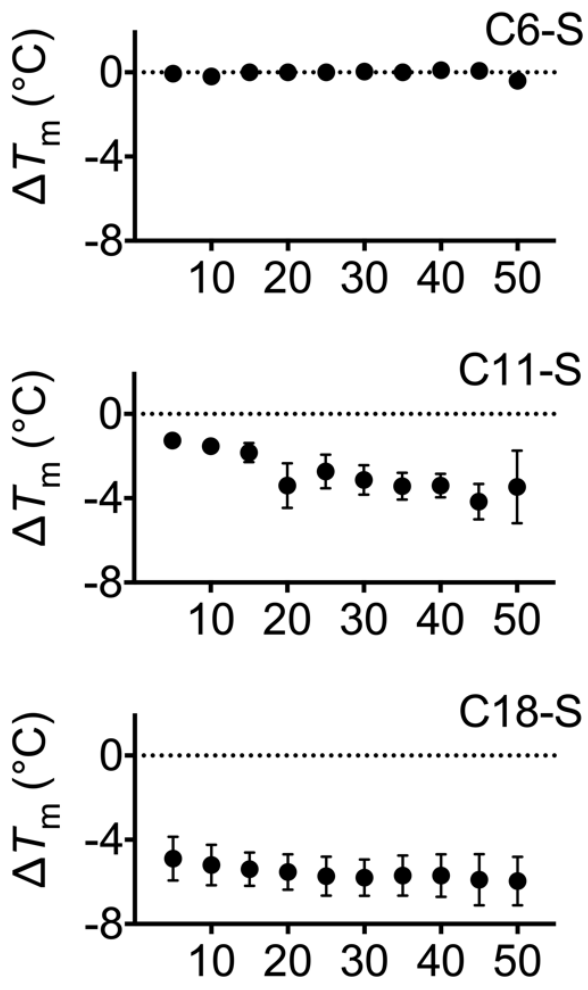

[alkyl sulfonate] $(\mu \mathrm{M})$
B.
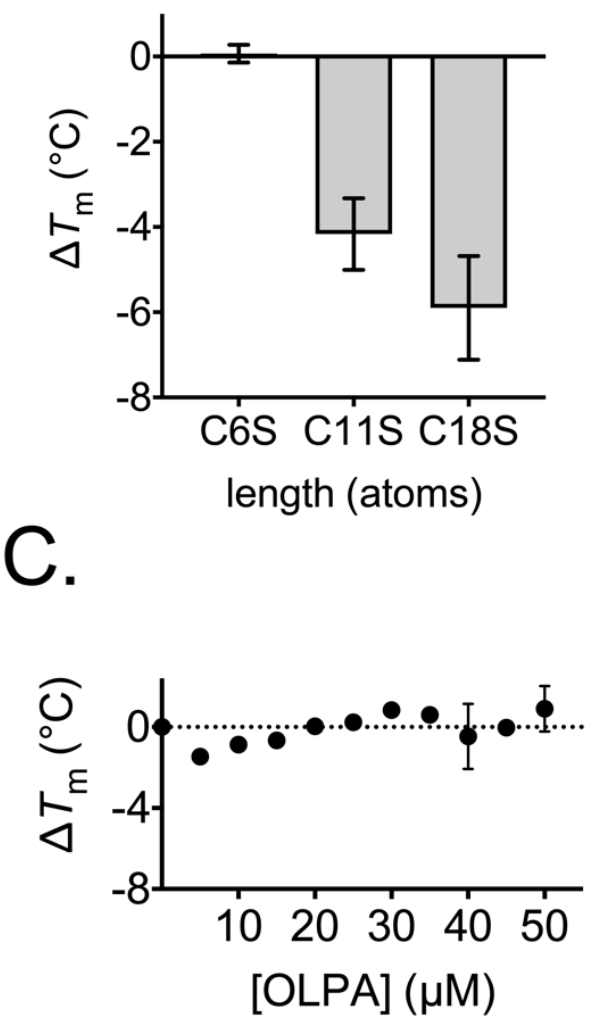

Figure 2. Alkyl sulfonate transport substrates and fatty acids induce the same specific destabilization of native UCP1. The relative thermal stability of purified UCP1 was determined as described for Fig. 1 (see Methods). The shifts in UCP1 thermal stability $\left(\Delta T_{\mathrm{m}}\right)$ associated with the addition of the indicated concentration of alkyl sulfonates (A) (C6-S, hexane sulfonate; C11-S, undecane sulfonate; C18-S, octadecane sulfonate; equivalent in atom length to C7C19, cf. Fig. 1), or at $25 \mu \mathrm{M}$ specifically (B), or with the addition of OLPA inhibitor (C), as indicated. Note, C18-S tests included $1.6 \mathrm{mM}$ methyl- $\beta$-cyclodextrin solubilizing agent. All values given are averages $( \pm S D)$ of 3 independent experiments. 

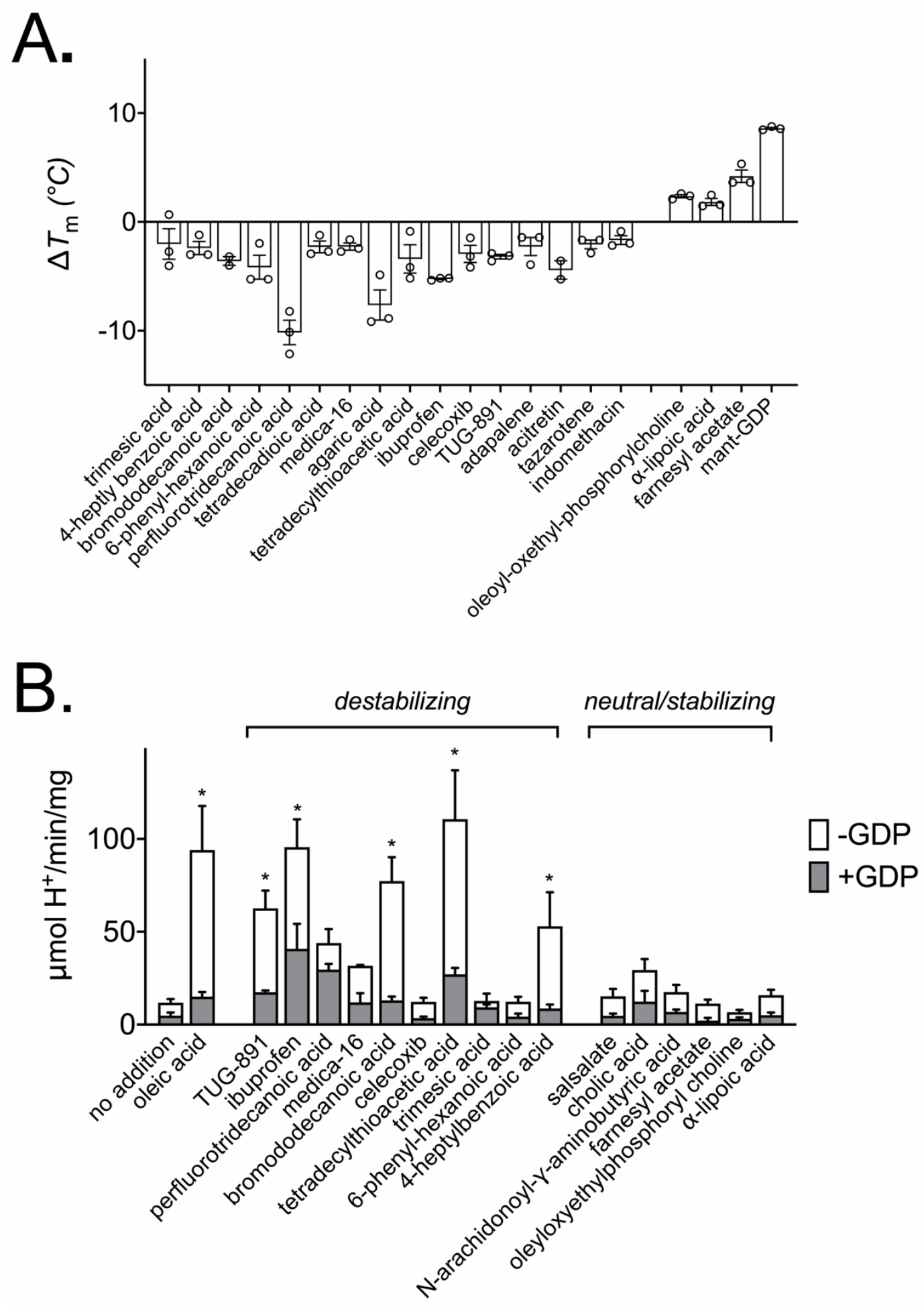

Figure 3. Novel activating ligands are found among compounds that destabilize UCP1 in stability screens. The relative thermal stability of purified UCP1 was determined as described for Fig. 1 (see Methods). A) Interacting ligands that induced a significant shift $\left(\Delta T_{\mathrm{m}}\right)$ in thermal stability of UCP1 following the screening of 72 notable compounds at $100 \mu \mathrm{M}$ (see Fig S2). Values are averages $( \pm S D)$ of 3 independent experiments. (B) The proton leak activity $(\mu \mathrm{mol}$ $\mathrm{H}^{+} / \mathrm{min} / \mathrm{mg}$ protein) by UCP1 in liposomes ( $\pm 1 \mathrm{mM}$ GDP) induced by selected compounds following thermostability shift analysis. Activators of proton leak are found specifically among compounds that were identified through a destabilizing interaction with UCP1. Values are averages ( \pm SEM) of 3-9 independent experiments. Statistical significance was determined by one-way ANOVA $\left({ }^{*} p<0.05\right)$. 
A.

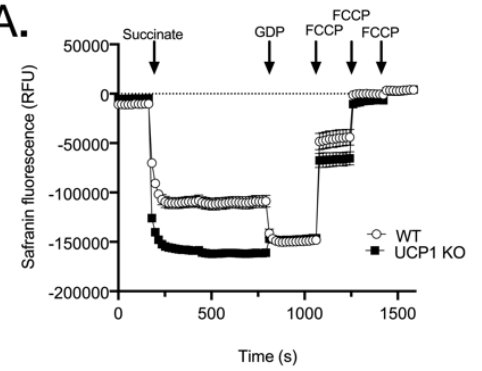

D.

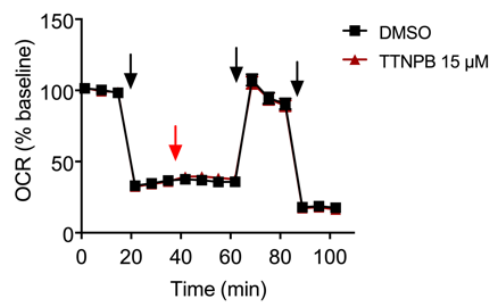

E.

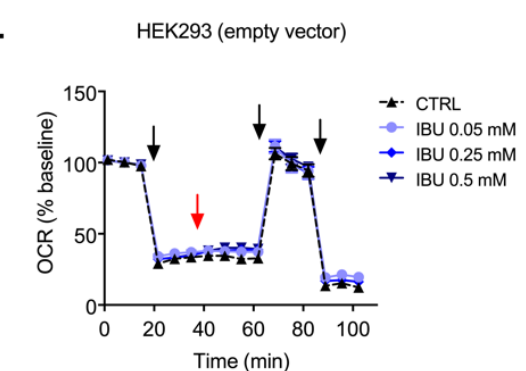

B.

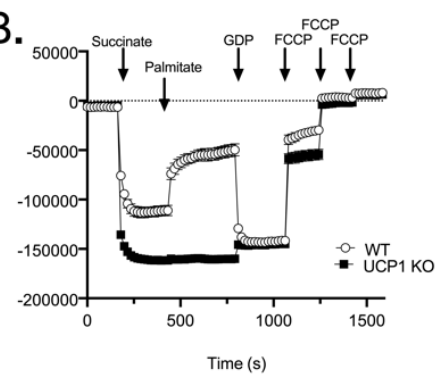

C.

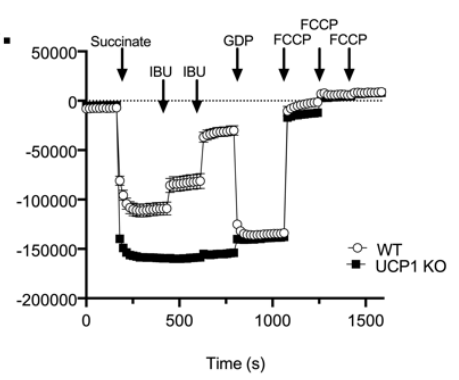

MmUCP1 HEK293

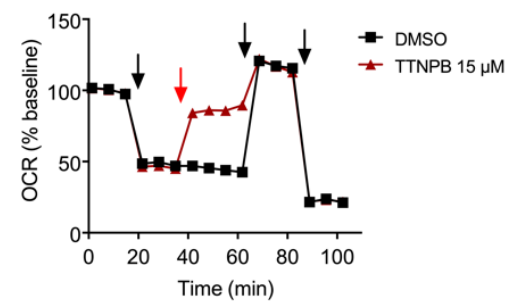

$\begin{array}{cl} & \square \text { CTRL } \\ \text { F. } & \square \text { IBU } 0.05 \mathrm{mM} \\ \text { IBU } 0.25 \mathrm{mM} \\ \text { IBU } 0.5 \mathrm{mM}\end{array}$

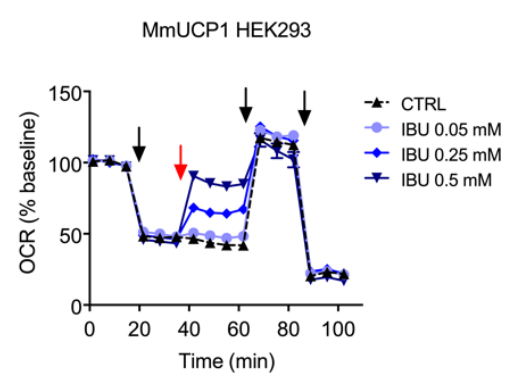

Figure 4. Ibuprofen stimulates UCP1 activity in mitochondria isolated from brown adipose tissue and in MmUCP1 (Mus musculus) transfected HEK293 cells. A-C: membrane potential measurements with safranin O for mitochondria isolated from wildtype (WT) or UCP1 KO mice, without $(A)$ or with additions of $100 \mu \mathrm{M}$ palmitate $(B)$ or $2 \times 250 \mu \mathrm{M}$ ibuprofen (C) and other effectors, as indicated (see SI Methods). $n=6$ samples per group, measured on two independent days. Results are shown as mean \pm SEM. ( $D$ and $E$ ) Oxygen consumption rate (OCR) of HEK293 cells transfected with an empty vector (EV) or mouse UCP1 (MmUCP1) upon treatment with TTNPB versus DMSO control (D) or different ibuprofen (IBU) concentrations versus buffer control $(E)$. Arrows indicate injections, which are 1. Oligomycin, 2. TTNPB or ibuprofen (red arrow), 3. DNP and 4. rotenone + antimycin A (see SI Methods). BSA concentration of medium: $0.4 \%$. (F) Summary of the OCR attributable to proton leak activity (oligomycin-insensitive respiration): treatment with ibuprofen stimulates UCP1 activity in a dose-dependent manner in MmUCP1 transfected HEK293 cells. Values ( \pm SEM) are from 17 to 23 samples per group, measured on four independent days, with statistical significance determined by two-way ANOVA $\left({ }^{*} p<0.05\right)$. 